\title{
SURDOSAGE ACCIDENTEL EN ALFATESINE CHEZ UN ENFANT POLYMALFORME
}

\author{
G. Boulard, G. Miet, J. Guerin et M. Sabathie
}

\author{
RÉSUMÉ
}

Un surdosage en alfatésine a été observé au cours d'une anesthésie pour craniosténose, chez un enfant polymalformé présentant un trouble majeur de la croissance ( $4 \mathrm{~kg}$ à 1 an) qui n'a pas pu être étiqueté. Le surdosage correspond à huit fois la posologie utile et cela pendant une période de trente minutes.

La seule conséquence nctable a été le retard du réveil justifiant la poursuite de la ventilation assistée. L'index thérapeutique de l'alfatésine rend compte de l'absence de conséquences graves.

L'ALFATÉsinE, anesthésique stéroïde intraveineux, est utilisée en anesthésiologie neuro-chirurgicale pour la qualité de l'anesthésie obtenue ainsi que pour son action favorable sur le débit et le métabolisme cérébral (diminution du débit et du métabolisme, avec baisse de la pression intra-crânienne). Un surdosage accidentel a été observé pendant la période d'entretien de l'anesthésie, chez un enfant de un an, pesant quatre kilogrammes, opéré d'une craniosténose dans un contexte polymalformatif complexe.

Cet enfant, dont la famille est originaire d'Afrique du Nord, est né au terme de 36 semaines, dysmature, au poids de $1270 \mathrm{~g}$. Le syndrome dysmorphique associe : oreilles décollées, mal ourlées et d'implantation basse, hypotélorisme, hirsutisme, microrétrognathisme, mal position des doigts de la main. A l'âge de deux mois, il ne pesait que $1500 \mathrm{~g}$ et présentait une intolérance alimentaire aux repas conventionnels (anticorps anti-lait de vache). Le diagnostic de leprèchaunisme a été évoqué mais non confirmć, et un trouble non étiqueté de la croissance n'a pu être mieux précisé. Le bilan hormonal complet est normal.

Il existe un caryotype $46 \mathrm{xy}$ et une cellule est porteuse de translocations (un des chromosomes de la paire $n^{\circ} 16$ et un de la paire $n^{\circ} 20$ ). Le fond d'ceil montre un aspect de choriorétinite. L’électro- encéphalogramme ne met pas en évidence de signe net de souffrance cérébrale, mais les différents potentiels évoqués montrent des anomalies de la maturation cérébrale. L'urographie intra-veineuse met en évidence un gros rein gauche avec distension calicielle sans distension de l'uretère, et un rein droit de taille nor-

G. Boulard, M. Sabathie, D.AR. III - (Pr. M. Sabathie); G. Miet, Service de Pédiatrie (Pr. C. Martin); J. Guerin, Clinique Neuro-Chirurgicale (Pr. P. Leman), Hópital Pellegrin, - 33076 Bordeaux, France.

$$
576
$$

male. Il n'existe pas de signe biologique d'insuffisance rénale. Enfin, l'examen radiographique du crâne montre une déformation symétrique du volume du crâne, avec débordement en longueur et en hauteur, contrastant avec un diamètre bi-pariétal rétréci, de nombreuses impressions digitiformes, et les sutures sagittales et coronales ne sont pas visibles. L'âge osseux se situe à cinq mois.

L'induction a été réalisée par $0,5 \mathrm{ml}$ d'alfatésine diluée à 10 pour cent, puis $50 \mu \mathrm{g}$ de fentanyl, curarisation, intubation et ventilation assistee par respirateur de Keiskamp (oxygène et protoxyde d'azote à 50 pour cent).

Les voies respiratoires supérieures étaient normales à l'examen en laryngoscopie directe et l'intubation a été réalisée sans aucune difficulté.

La dose d'entretien théorique était de $0,125 \mathrm{ml} \cdot \mathrm{kg}^{-1} \cdot \mathrm{h}^{-1}, c^{\prime}$ est-à-dire de $0,5 \mathrm{ml} \cdot \mathrm{h}^{-1}$ d'alfatésine, administrée par perfusion à débit constant. Le surdosage s'est produit au décours de l'induction et s'est prolongé pendant toute la durée de l'installation du patient en position opératoire (position semi assise et hyperflexion de la tête). Il est la conséquence d'une erreur dans le réglage du régulateur de débit.

Ainsi, l'enfant reçoit accidentellement $4 \mathrm{ml}$ d'alfatésine en 30 minutes, c'est-à-dire 16 fois la dose théorique pour la même période. Comptetenu de la posologie moyenne d'entretien nécessaire chez l'enfant de moins de $10 \mathrm{~kg}(0,253$ $\left.\mathrm{ml} \cdot \mathrm{kg}^{-1} \cdot \mathrm{h}^{-1} \pm 0,073\right)^{2}$ le surdosage peut être estimé à huit fois la posologie utile. L'administration d'alfatésine est alors interrompue. La seule manifestation contemporaine du surdosage est une chute de pression artérielle systolique de 90 à $75 \mathrm{~mm} \mathrm{Hg}$. La fréquence cardiaque ne s'est pas modifiée. En fin d'intervention, la température rectale est à $36^{\circ} \mathrm{C}$, mais le réveil sera retardé de 110 minutes. Cette phase exigera la poursuite 
de la ventilation assistée avant de voir réapparaître une respiration spontanée avec pauses, puis un réveil progressivement complété autorisant l'extubation. Cet accident n'aura aucune conséquence apparente pendant les semaines durant lesquelles l'enfant a été suivi.

Cette observation de surdosage chez un enfant polymalformé présentant un trouble majeur de la croissance, est différente de celle de BoggildMadsen'. Il s'agissait d'un adulte ayant reçu $35 \mathrm{ml}$ d'Alfatésine pure en deux minutes, sans conséquence hémodynamique, seule une hypothermie post-opératoire importante $\left(31^{\circ} \mathrm{C}\right)$ était notée. Expérimentalement, chez la souris, l'index thérapeutique (DL 50/DE 50) est de 30,4 pour l'alfatésine contre 5,9 à 8,5 pour thiopentone, propanidide et kétamine (Davis cité par Boggild-Madsen).' L'absence de conséquence du surdosage est imputable à l'index thérapeutique élevé du produit, alors qu'un tel surdosage avec un autre produit anesthésique entraînerait des conséquences dramatiques. Dans l'observation rapportée, seul le réveil sera considérablement retardé, et il n'a pas été retrouvé d'hypothermie notable.

\section{BIBLIOGRAPHIE}

1. Boggild-Madsen, N.B. \& Cargnelli, T. Accidental over-dose of alfathesin under general anaesthesia: case report. Canad. Anaesth. Soc. J. 25: 245-246(1978).

2. Boulard, G., NiCod, J., SCOntrini, G. \& Sabathie M. Neuroanesthésiologie chez l'enfant de moins de dix kilos : intérêts de l'alfatésine associée à un morphinomimétique. Ann. Anesth. Franç. - A paraître.

\section{ABSTRACT}

An overdosage of alfathesin was accidentally administered to a $4 \mathrm{~kg}$ infant with multiple congenital anomalies during an operation for craniosynostosis. The overdose was a consequence of a mistake in adjusting the rate of administration of a constant flow infusion pump. The total dosage corresponded to eight times the necessary dose administered over a period of 30 minutes.

The only notable effect was a delayed awakening, which necessitated assisted ventilation. The high therapeutic index of alfathesin is responsible for the absence of serious consequences. 